and Home Blood Pressure Monitoring Trial (e-BP). Patients were asked whether they took their BP at home. Patients with essential HTN willing to participate were invited to 2 screening visits to determine if they had uncontrolled BP and eligible for study participation. We describe here the relationship between HBPM and willingness to attend a screening visit, and for those who had a screening visit whether prior use of HBPM predicted BP control. Results: Of the 9298 patients sampled, 8840 (95.1\%) were contacted, and 7268 (82.2\% of contacted) answered the HBPM question, with $60.8 \%$ stating they used a HBPM. After excluding 2684 participants who did not meet study eligibility criteria, patients who were eligible but who refused to attend a screening visit $(n=2,078)$ were more likely to use HBPM than those willing to attend the screening visit $(\mathrm{n}=2,506)(67.9 \%$ vs. $57.9 \%$, $P=<.001)$. Among patients with a BP measure at the first screening visit, those who reported using HBPM trended toward improved BP control (BP $<140 / 90 \mathrm{~mm} \mathrm{Hg}$ ) compared to patients not using HBPM, however this was relationship was not statistically significant $(53.2 \%$ vs. $50.5 \%, \mathrm{p}=0.18)$. Discussion: Hypertensive patients who were not willing to participate in a study to use HBPM and Web communications to improve BP control were more likely to already use HBPM. Prior use of HBPM trended towards improved BP control, but not significantly. HBPM use by itself does not predict HTN control, possibly because people may use these for different reasons.

Keywords: Home blood pressure monitoring, Hypertension, Prevention and control

doi:10.3121/cmr.2011.1020.ps2-14

C-A4-03:

Medication Adherence in Patients with Uncontrolled Blood Pressure: Results from the Hyperlink Study

Simrandeep Tiwana, $\mathrm{PhD}^{1}$; Tessa Kerby, $\mathrm{BA}^{1}$; Stephen Asche, $\mathrm{MA}^{1}$; Nicole Schneider, $\mathrm{BS}^{1}$; Pamala Pawloski, PharmD ${ }^{1}$; Michael Maciosek, $\mathrm{PhD}^{1}$; Sarah Groen, PharmD ${ }^{1}$; Holly Kadrmas, PharmD ${ }^{1}$; Krissa Klotzle, PharmD ${ }^{1}$; Ryan Michels, PharmD ${ }^{1}$; Karen Margolis, MD, $\mathrm{MPH}^{1}$

${ }^{1}$ HealthPartners

Background/Aims: The Hyperlink study is a cluster randomized trial in patients with uncontrolled blood pressure. The objectives of this analysis were: (a) to compare measures of self reported adherence with actual adherence to medications at study enrollment (b) to study predictors of adherence. Methods: We analyzed prescription claims data for 146 participants who had prescription coverage through HealthPartners for at least one year prior to study enrollment and were taking at least 1 antihypertensive medication. Self reported adherence was measured using a 4-item Morisky scale. Actual adherence was defined as a continuous measure of Medication Possession Ratio (MPR) as well as a binary measure defining adherence as an MPR of $>80 \%$. Results: Of the 146 study participants, mean age was 66 (37-89 years), 14\% were non-white, $48 \%$ were females, $45 \%$ had a $4 \mathrm{yr}$ college degree. Mean MPR was $0.86,71 \%$ of patients were adherent by the binary MPR measure, and 70\% self-reported high adherence (Morisky score $=0$ ). Higher self-reported adherence (lower Morisky score) was significantly associated with higher MPR (Pearson $\mathrm{r}=-0.4 ; \mathrm{p}<0.001$ ). Results of multiple linear regression showed that higher age $(p=.04)$ and higher number of medications $(\mathrm{p}=0.04)$ predicted higher mean MPR. Race, gender and education were not associated with MPR. Higher self-reported adherence was associated with higher age but not race, gender, education, or number of medications. Conclusions: Self reported adherence was correlated with actual adherence in this sample of patients with uncontrolled blood pressure. Age and number of medications were important predictors of adherence. Our finding that patients on multiple drugs tend to be more adherent differs from many other studies, and may be related to the study population being sufficiently motivated to volunteer for a trial.

Keywords: Adherence, Hypertension, Medication

doi:10.3121/cmr.2011.1020.c-a4-03
PS2-07:

The Association of Cyberbullying with Cardiovascular Health in Adolescents: A Preliminary Analysis

Andrea Cassidy-Bushrow, PhD, $\mathrm{MPH}^{1}$; Dayna Johnson, MSW, $\mathrm{MPH}^{1}$; Christine Joseph, $\mathrm{PhD}, \mathrm{MPH}^{1}$

${ }^{1}$ Henry Ford Health System

Background/Aims: With increasing access to the internet and other technology, adolescents may become victims of online harassment, referred to as cyberbullying. Cyberbullying may cause worry, fear, and distress among youth, all which increase cardiovascular disease (CVD) risk in adults. To our knowledge, no study has examined the potential association of cyberbullying with CVD risk factors in adolescents. We examined the association of cyberbullying with overweight/obesity and elevated blood pressure (BP) among healthy adolescents. Methods: Adolescents age 14-17 years and parent/guardian were invited to a research visit between November, 2009 and present. Height, weight, and BP were measured by trained staff and internet experiences quantified by questionnaire. Race was defined as African-American or other race. Cyberbullying was defined as self-report in the past year of being worried or threatened because of being bothered or harassed online or of being embarrassed by others online. Overweight/ obesity was defined as BMI $>=85$ th percentile for gender and age. Elevated BP was defined as a SBP or DBP $>=90$ th percentile for gender, age and height. Logistic regression models were fit to estimate the association of cyberbulling with overweight/obesity or elevated BP. Results: As of October, 2010, 190 adolescents with complete data have been recruited into the study. Mean age was $16.5 \pm 1.0$ years; $76(40 \%)$ were male and $112(59.0 \%)$ were African-American. Mean BMI of adolescents was $24.2 \pm 6.4 \mathrm{~kg} / \mathrm{m}^{\wedge} 2$ and mean SBP and DBP were $117.7 \pm 11.3 \mathrm{mmHg}$ and $63.9 \pm 7.1 \mathrm{mmHg}$, respectively; 62 (32.6\%) were classified as overweight/obese and $28(14.7 \%)$ had an elevated BP. A total of $29(15.3 \%)$ adolescents reported being cyberbullied; older adolescents $(P=0.061)$ were more likely and AfricanAmericans $(P=0.040)$ were less likely to report being cyberbullied. Gender was not associated with cyberbullying $(P=0.143)$. Cyberbullying was not associated with overweight/obesity $(P=0.951)$ or elevated BP $(P=0.701)$, after adjustment for other risk factors. Conclusions: CVD risk factors in youth track into adulthood, thus early-life experiences represent important and potentially modifiable predictors of adult disease. In this study, selfreported experiences of cyberbullying were not associated with being overweight/obese or with elevated BP. Continued study of psychosocial risk factors, including cyberbullying, with adolescent CVD health is warranted. Keywords: Cyberbullying, Adolescent, Cardiovascular doi:10.3121/cmr.2011.1020.ps2-07

\section{Child Health}

C-B5-04:

Patterns of Blood Pressure Measurement at Pediatric Primary Care Visits in Large Medical Group Practices

Emily Parker, $\mathrm{PhD}, \mathrm{MPH}^{1}$; Patrick O'Connor, MD, $\mathrm{MPH}^{1}$; Kenneth Adams, $\mathrm{PhD}^{1}$

\section{${ }^{1}$ HealthPartners Research Foundation}

Background/Aims: Current estimates suggest that high blood pressure is present in $3-5 \%$ of children, although the prevalence of hypertension in obese children may be as high as $20 \%$. The American Academy of Pediatrics recommends routine blood pressure measurement in children over the age of 3. However, it is not known whether this recommendation is being followed or what factors influence blood pressure measurement in pediatric populations. Methods: We examined 115,695 pediatric patients ages 3-17 who had an outpatient primary care visit (family practice or pediatrics) between 2007 and 2009 in two large medical groups in Minnesota and Colorado. Patient characteristics (sex, age, BMI percentile, and race/ethnicity), and clinic department were extracted from electronic medical record data. The predicted probability of blood pressure measurement was estimated using binomial mixed-model logistic regression with maximum likelihood estimation 
adjusted for sex, age, BMI percentile, race/ethnicity and clinic department. Results: Overall, blood pressure was measured at $>80 \%$ of children's visits to pediatric and family medicine clinics. The predicted probability of a blood pressure recorded at a primary care visit increased steadily with age (from .81 in 3-5 year olds to .89 in 15-17 year olds, $p$ for trend $<.0001$ ). Of the $60 \%$ of visits where children had a recorded BMI, children with a BMI $=95$ th percentile were slightly less likely to have a blood pressure recorded compared to BMI 85 th $<95$ th, and BMI $<85$ th $(91 \%, 93 \%, 94 \%$, respectively, $\mathrm{p}<.0001)$. There were no differences by sex or race/ethnicity in the adjusted prevalence of documented blood pressure measurement. Conclusions: Blood pressure measurements are documented at a large proportion of children's visits to pediatric and family medicine clinics. Younger children were less likely to have blood pressure measurements recorded than adolescents. The relative lower likelihood of blood pressure measurement in obese children warrants further investigation given the higher prevalence of hypertension in these children.

Keywords: Pediatric, Hypertension, Obesity

doi:10.3121/cmr.2011.1020.c-b5-04

C-B5-03:

Linking Parent and Child Medical Records to Identify Youth at Risk for Familial Hypercholesterolemia

Catherine McNeal, $\mathrm{MD}, \mathrm{PhD}^{1}$; Debbie Godwin, $\mathrm{BS}^{1}$; Linda Robinson, BS, $\mathrm{MA}^{1}$; Dean Kjar, $\mathrm{MS}^{1}$; Bhushan Mahajan, $\mathrm{MS}^{1}$; Teresa Ponder ${ }^{1}$; Anne Robertson ${ }^{1}$

\section{${ }^{1}$ Scott \& White Healthcare}

Background: Despite consistent recommendations in numerous guidelines sponsored by professional societies, screening youth for familial hypercholesterolemia $(\mathrm{FH})$ and/or those with a family history of premature cardiovascular disease (CVD) identifies only a small percentage of high-risk youth. By linking parent and child data in the virtual data warehouse, we can assess current screening practices, factors that impact screening practices and ultimately strategies aimed at improving childhood screening. Aims: the aim of this study is to compare cholesterol testing and screening rates in youth who have at least one parent covered under the same health plan with a history of CVD defined as a history of myocardial infarction, percutaneous coronary artery intervention (PCI) or coronary artery bypass surgery (CABG) and/or a parent with FH established by ICD-9 diagnoses or an abnormal cholesterol level. Methods: A cohort of youth who were 2-26 years of age anytime between service dates inclusive of 01/01/2001 and 12/31/2009 and who were covered as insured members under their parents' health plans within the Scott \& White HMO were identified including a subset with any ICD-9 diagnosis code associated with hyperlipidemia. Parent-child linkagages were created using a subscriber_id relationship code and relationship description. The parental cohort with CVD was identified from the CVRN Surveillance study as well as parents who have FH defined as an LDL-C of $>215 \mathrm{mg} / \mathrm{dl}$ or a total cholesterol $>300 \mathrm{mg} / \mathrm{dl}$. Results: We found that adherence to guidelines for cholesterol screening of youth at a higher risk for premature CHD is no greater than screening rates for children with a lower risk who do not have an affected parent. Moreover, a family history of CHD and hypercholesterolemia is rarely documented in a dependent's medical record. Conclusions: Cost-effective approaches to screening for FH in the pediatric population rely on a parental history of CVD and/or FH. However, this approach does not identify the majority of youth at risk for premature CVD. Universal screening of youth may be warranted.

Keywords: Hypercholesterolemia, Children, Screening doi:10.3121/cmr.2011.1020.c-b5-03

C-B5-01:

Childhood Obesity: Risk Factors, Associated Morbidity and Service Utilization

Revital Gross, $\mathrm{PhD}^{1}$; Daphna Doron, MD, $\mathrm{MHA}^{2}$; Yael Ashkenazi, $\mathrm{MPH}^{1}$; Beatriz Hemo, $\mathrm{MA}^{2}$; Orit Ben Shoham, MHA ${ }^{2}$; Israela Nahshon, MHA ${ }^{2}$

${ }^{1}$ Myers-JDC-Brookdale Institute; ${ }^{2}$ Maccabi Healthcare Services
Aims: To identify parent-level and child-level risk factors for childhood obesity, to study health consequences and medical service utilization among the overweight children. Methods: The study included families with children aged 4-11 years old, registered with Maccabi Healthcare Services at 2 community clinics in the Greater Jerusalem Area. During 2008, 409 mothers completed questionnaires and interviewers weighed and measured 714 children. There was a $50 \%$ response rate. Information on associated morbidity and utilization of Health Services was obtained from Maccabi's computerized database. Results: Approximately 30\% (122 of 409) of the families had at least one overweight or obese child (BMI $>85$ th percentile / BMI $>95$ th percentile, respectively). Twenty percent of the children sampled were overweight or obese(overweight: $11.9 \%, 85$ of 714 . obese: $8.3 \%, 59$ of 714). The variables with an independent effect on having at least one overweight child in the family were: father of Asian-African origin; immigration to Israel since 1995; non-academic education; a maternal age less than 40; a mother who had a history of being overweight and who reported lack of control over her eating habits. Variables with an independent effect on children's obesity/overweight were: age below 7 years old; eats a broad variety of foods (not fussy); has an 'inactive' lifestyle; eats the main daily meal at a daycare center or afternoon enrichment center at least once a week. Among the overweight children, mainly in the 7-11 age groups, a higher rate of associated morbidity (particularly asthma, orthopedic problems and dermatological conditions) was documented. The service utilization rates were also higher among overweight children compared to children within the normal weight range. Conclusions: Pro-active medical monitoring of overweight or obese children must be encouraged even if they do not present other symptoms. Parents and caregivers should be provided with information regarding the risk factors for obesity, as some of those are modifiable. It is recommended that a follow-up study be conducted with a national representative sample of children to verify the findings.

Keywords: Obesity, Child, Risk factors

doi:10.3121/cmr.2011.1020.c-b5-01

\section{C-B5-02:}

A Parent Failure Modes and Effect Analysis: Parent Teams Can Identify Problems and Propose Interventions

Joann Wagner, MSW ${ }^{1}$; Kathleen Walsh, MD, $\mathrm{MSc}^{2}$; Juliet Moretti, $\mathrm{BA}^{1}$; Terry Field, DSc ${ }^{1}$; Kathleen Mazor, Ed, DMS ${ }^{1}$; Jennifer Donovan, PharmD ${ }^{3}$; Abir Kanaan, PharmD ${ }^{3}$

${ }^{1}$ Meyers Primary Care Institute-UMass Medical School, Fallon Clinic, FCHP; ${ }^{2}$ UMass Medical School, Meyers Primary Care Institute; ${ }^{3} \mathrm{MCPHS}$, Meyers Primary Care Institute

Background: Parents of many children with cancer administer highly complex chemotherapy regimens at home which are error prone. One way to identify and repair potential error prone processes is with a Failure Mode and Effect Analysis (FMEA). An FMEA is a systematic, team-based approach to understanding the ways a process can fail and developing interventions; it has been used in hospitals for decades but has never, to our knowledge, been used with a team of parents. Methods: The objective of this study was to identify aspects of the home medication use processes at risk for error and propose interventions using an FMEA with a group of parents. We recruited parents of children with cancer taking home medications. FMEA steps included: selection of a high-risk process; diagram the process; brainstorm potential failure modes; rate and prioritize failure modes; identify root causes; and redesign the process. The target high-risk process was parent/ caregiver administration of 6-mercaptopurine following a change in medication dose. Parents were trained in FMEA methodology using materials at a 6th grade reading level and a real-life example. Failure modes were prioritized using ratings for severity, frequency of occurrence, and detectability. Results: The process diagram developed by the parent-team included 12 steps. The highest priority failure modes were: (1) shared instructions by the parent who attended the clinic visit regarding change in dose are misunderstood by the other caregiver(s); (2) administration of chemotherapy without realizing that another caregiver already administered it; (3) parent misunderstanding of physician's instructions about the change in dose; and (4) the parent who attended the clinic visit shares incorrect instructions regarding the change in dose with other caregivers. During the 